\title{
Formation of Lipofuscin-Like Autofluorescent Granules in the Retinal Pigment Epithelium Requires Lysosome Dysfunction
}

\author{
Cristina Escrevente, ${ }^{1}$ Ana S. Falcão, ${ }^{1}$ Michael J. Hall, ${ }^{2}$ Mafalda Lopes-da-Silva, ${ }^{1}$ Pedro Antas, ${ }^{1}$ \\ Miguel M. Mesquita, ${ }^{1}$ Inês S. Ferreira, ${ }^{1}$ M. Helena Cardoso,${ }^{1}$ Daniela Oliveira, ${ }^{1}$ \\ Ana C. Fradinho, ${ }^{1}$ Thomas Ciossek, ${ }^{3}$ Paul Nicklin, ${ }^{3}$ Clare E. Futter, ${ }^{2}$ Sandra Tenreiro, ${ }^{1}$ and \\ Miguel C. Seabra ${ }^{1,2}$ \\ ${ }^{1}$ iNOVA4Health, CEDOC - Chronic Diseases Research Center, NOVA Medical School, Universidade Nova de Lisboa, Lisboa, \\ Portugal \\ ${ }^{2}$ UCL Institute of Ophthalmology, London, United Kingdom \\ ${ }^{3}$ Research Beyond Borders, Boehringer Ingelheim, Biberach, Germany
}

Correspondence: Miguel C. Seabra, CEDOC - NOVA Medical School, Universidade Nova de Lisboa, 1169-056 Lisboa, Portugal; miguel.seabra@nms.unl.pt.

Received: January 24, 2021

Accepted: June 29, 2021

Published: July 27, 2021

Citation: Escrevente C, Falcão AS, Hall MJ, et al. Formation of lipofuscin-like autofluorescent granules in the retinal pigment epithelium requires lysosome dysfunction. Invest Ophthalmol Vis Sci. 2021;62(9):39.

https://doi.org/10.1167/iovs.62.9.39
Purpose. We aim to characterize the pathways required for autofluorescent granule (AFG) formation by RPE cells using cultured monolayers.

MEthods. We fed RPE monolayers in culture with a single pulse of photoreceptor outer segments (POS). After 24 hours the cells started accumulating AFGs that were comparable to lipofuscin in vivo. Using this model, we used a variety of light and electron microscopical techniques, flow cytometry and Western blot to analyze the formation of AFGs. We also generated a mutant RPE line lacking cathepsin D by gene editing.

Results. AFGs seem to derive from incompletely digested POS-containing phagosomes and after 3 days are surrounded by a single membrane positive for lysosome markers. We show by various methods that lysosome-phagosome fusion is required for AFG formation, and that impairment of lysosomal $\mathrm{pH}$ or catalytic activity, particularly cathepsin $\mathrm{D}$ activity, enhances AF accumulation.

Conclusions. We conclude that lysosomal dysfunction results in incomplete POS degradation and enhanced AFG accumulation.

Keywords: retinal pigmented epithelium, photoreceptor outer segments, autofluorescent granules, lipofuscin, lysosome dysfunction
A MD is the most common blinding disease in the Westthe retina, which impacts the quality of life of the elderly. Currently, there are no effective therapies for the common forms of AMD namely early, intermediate, or late stage "dry" AMD, also called geographic atrophy. ${ }^{1,2}$ The primary cause of pathology in AMD seems to be RPE degeneration. RPE thinning and depigmentation leads to atrophy and the accumulation of extracellular deposits called drusen between the RPE and the choroid. ${ }^{3}$ Eventually, RPE atrophy leads to photoreceptor loss. In fact, photoreceptors, responsible for phototransduction, rely on the RPE for nutrients and waste disposal.

The RPE is responsible for the daily digestion of photoreceptor outer segments (POS), a process essential for sustained photoreceptor function which requires the regular recycling of visual cycle components. ${ }^{4}$ However, POS digestion by RPE cells imposes a continuous heavy burden on the lysosomal network of these nondividing cells. Another AMD hallmark is the accumulation of lipofuscin in the RPE., 6 The appearance of lipofuscin, clinically detected as fundus autofluorescence (AF) occurs naturally and accumu- lates gradually with age, but it is exacerbated in disease, being a predictive marker of retinal stress. ${ }^{7-9}$

The age-related pigment lipofuscin is particularly abundant in nerve cells, cardiac muscle cells and skin. Accordingly, its origin as well as its composition vary among tissues, being mainly constituted by oxidized proteins $(30 \%-70 \%)$, lipids $(20 \%-50 \%)$, metal cations (2\%), and sugar residues. ${ }^{10}$ These differences in composition are reflected in very wide spectra of lipofuscin fluorescence emission ranging from 400 to $700 \mathrm{~nm} .{ }^{11}$ Lipofuscin accumulates in lysosomes and for many cell types, is thought to originate internally resulting from altered cellular proteostasis, autophagy, and mitophagy and/or dysfunction of lipid metabolism. ${ }^{10}$ Lipofuscin in the RPE is proposed to be a byproduct of POS digestion, and the final maturation step of ingested phagosomes is fusion with lysosomes. ${ }^{8,12-14}$

Lysosomes are critical players in the complex cellular network that the comprises endolysosomal, phagocytic, and autophagic pathways, coupled with metabolic sensing. ${ }^{15}$ Lysosome involvement in phagosome degradation within the RPE was initially described by electron microscope (EM) studies that identified acid phosphatase activity in both 
lysosomes and POS phagosomes. ${ }^{13,14}$ Over the years, multiple studies have demonstrated that POS phagosome degradation is a very complex multistep process, involving different hydrolases and the coordination of different molecular motors (reviewed in $^{4}$ ).

The heavy, daily, phagocytic load on the RPE, together with a highly oxidative environment and high metabolic activity, leads to the slow accumulation of indigestible material over decades. The inability to fully digest POS and the lifelong accumulation of undigested material led to the idea that lysosomal dysfunction in the RPE is at the center of a pathogenic hub contributing to proteotoxicity, mitochondrial dysfunction, redox imbalance, and inflammation in AMD, eventually leading to RPE cell death and vision loss. ${ }^{16}$ More generally, evidence indicates that the lysosomal network is prone to age- and disease-related dysregulation, including in neurodegenerative diseases like Parkinson disease. ${ }^{17,18}$

The formation and maintenance of lipofuscin within RPE cells remains poorly characterized. In this work, we have optimized an in vitro model system that recapitulates AMD features. Feeding RPE cells with a single pulse of porcine POS leads to accumulation of autofluorescent granules (AFGs) similar to lipofuscin in vivo. ${ }^{19-25}$ Our results suggest that some lysosome activity, in particular lysosome-phagosome fusion, is required for AFG formation, but that impairment of lysosome catalytic activity, particularly cathepsin D (CTSD) activity, enhances AFG accumulation. Therefore, dysfunctional lysosomal activity leads to decreased POS degradation and increased AF accumulation.

\section{METHODS \\ Cell Cultures}

Primary human fetal RPE cells (hfRPE) were purchased from Lonza and cultured using optimized RtEBM Basal Medium and RtEGM SingleQuots Supplements (Lonza Group AG, Basel, Switzerland), according to the supplier's instructions. For confocal or EM assays, cells were seeded on laminin-coated (BioLamina, Sundbyberg, Sweden) coverslips $(10 \mu \mathrm{g} / \mathrm{mL})$. Cells were used after 21 days in culture, after which they demonstrate a cobblestone morphology with the formation of tight junctions confirmed by transepithelial electric resistance values of $\pm 250 \Omega . \mathrm{cm}^{2}$, measured using an electrode (STX2; World Precision Instruments, Sarasota, FL). ARPE-19 cells (ATCC, Manassas, VA) were cultured in Dulbecco's Modified Eagle Medium/Nutrient Mixture F-12 (DMEM/F-12) (Gibco, Grand Isle, NY) supplemented with $10 \%$ fetal bovine serum (FBS) (Gibco) and 1\% penicillinstreptomycin (Pen/Strep) (Gibco). For porcine RPE (pRPE) primary cultures, porcine eyes were collected from a slaughterhouse, kept on ice, and the procedure for cell culture was performed on the same day. Briefly, external tissue from the eyeballs was removed and eyes were cleaned in an iodine surgical scrub solution, diluted 1:4 in water. Eyes were washed in a Pen/Strep/PBS solution for 5 minutes. The eyes were opened, inside a class II biosafety cabinet, using a scalpel, and kept in multiwell dishes, opening facing up. The eye cups were filled with PBS and the neural retinas were removed. PBS was replaced by trypsin and the eye cups were incubated for 30 minutes, at $37^{\circ} \mathrm{C}$. The RPE was resuspended in Dulbecco's Modified Eagle Medium: Nutrient Mixture F-12 (DMEM/F-12; Gibco) supplemented with $1 \%$ sodium pyruvate, $1 \%$ nonessen- tial amino acids (both from Biowest, Riverside, MO), 1\% Pen/Strep, and $1 \%$ FBS, spun at $200 \times g$ at room temperature, 5 minutes. The pelleted cells were resuspended in fresh medium (containing 10\% FBS) and seeded in six-well plates. This medium is changed once a week until cells reach confluency, at which point they are changed to a reduced serum medium (1\% FBS). Primary cultures can be expanded and used for assays, by plating onto multiwell plates. Whenever using glass coverslips or transwells, cells were seeded on laminin-coated (BioLamina) coverslips $(10 \mu \mathrm{g} / \mathrm{mL})$. All of the cell models were grown in a $5 \% \mathrm{CO}_{2}$ incubator at $37^{\circ} \mathrm{C}$.

\section{POS Isolation and Gold Tagging}

POS were isolated from porcine eyes, according to ${ }^{26}$ with some minor modifications. Briefly, porcine eyes were collected from a slaughterhouse and kept on ice. External tissue from the eyeballs was removed and eyes were washed in a 1\% Pen/Strep in PBS solution for 5 minutes. The eyes were opened, using a scalpel, and kept in multiwell dishes, opening facing up. The eye cups were filled with PBS and the neural retinas were removed and collected in a falcon containing homogenization solution (20\% sucrose, $20 \mathrm{mM}$ tris acetate $\mathrm{pH} 7.2,2 \mathrm{mM} \mathrm{MgCl}_{2}, 10 \mathrm{mM}$ glucose, $5 \mathrm{mM}$ taurine). Retina homogenate was filtered through gauze and added to a continuous sucrose gradient $(25 \%-$ $60 \%$ sucrose, $20 \mathrm{mM}$ tris acetate $\mathrm{pH} 7.2,10 \mathrm{mM}$ glucose, 5 $\mathrm{mM}$ taurine). POS were separated by centrifugation at 25.000 $\mathrm{rpm}(106.750 \times \mathrm{g})$ for 120 minutes, in a swing rotor (SW-32Ti; Beckman, Pasadena, CA). A single orange band in the upper one-third of the gradient, corresponding to POS, was aspirated with a P1000 tip and collected into a new falcon. POS were washed three times (wash 1:20 mM tris acetate $\mathrm{pH}$ 7.2, $5 \mathrm{mM}$ taurine; wash 2: $10 \%$ sucrose, $20 \mathrm{mM}$ tris acetate $\mathrm{pH}$ 7.2, $5 \mathrm{mM}$ taurine; wash 3: $10 \%$ sucrose, $20 \mathrm{mM}$ sodium phosphate $\mathrm{pH} 7.2,5 \mathrm{mM}$ taurine) by sequentially centrifugations $\left(5000 \mathrm{rpm}\right.$ for 10 minutes at $\left.4{ }^{\circ} \mathrm{C}\right)$ and resuspension of the pellet. POS preparations were washed in PBS and resuspended in medium and protein content was measured (BCA Protein Assay Kit, Thermo Scientific, Waltham, MA) and in parallel, POS particles were counted in a cell counting chamber POS then were aliquoted and stored at $-80{ }^{\circ} \mathrm{C}$, in medium containing $10 \% \mathrm{FBS}, 2.5 \%$ sucrose, $0.04 \%$ sodium azide, and $1 \%$ Pen/Strep.

For the UV-irradiated POS, each prep was exposed to ultraviolet radiation using a UVP Crosslinker (CL-1000 Model, Analytik Jena, Jena, Germany) with $2 \times 2$ minutes pulses of $254 \mathrm{~nm}$ at an estimated radiant exposure of $1 \mathrm{~J} / \mathrm{cm}^{2}$.

For gold tagging, POS preps were thawed, pelleted, and resuspended in $200 \mu \mathrm{L}$ of $10 \mathrm{~nm}$ gold colloid solution (BBI Solutions, Portland, ME). After a mild sonication for 10 minutes in a sonicator bath, POS were washed three times in PBS.

\section{POS Phagocytosis Assays}

Before the phagocytosis assays, POS preps were thawed in the dark, washed three times in PBS by spinning for 10 minutes at $2.400 \times g$. RPE cells were fed during 4 hours with POS at a final concentration of $50\left(6.5 \times 10^{4}\right.$ POS particles $/ \mathrm{cm}^{2}$; approximately $2.5 \times 10^{5}$ POS particles $/ \mathrm{mL}$ ) or $200 \mu \mathrm{g} / \mathrm{mL}$ (approximately $2.6 \times 10^{5}$ POS particles $/ \mathrm{cm}^{2}$; approximately $1 \times 10^{6}$ POS particles $/ \mathrm{mL}$ ) in Dulbecco's Modified Eagle Medium: Nutrient Mixture F-12 
(DMEM/F-12; Gibco) supplemented with 1\% sodium pyruvate, $1 \%$ nonessential amino acids (both from Biowest), $1 \%$ Pen/Strep, and 10\% FBS. After this pulse (time 0 hour), POS were removed, cells were washed and cultured in supplemented DMEM with 1\% FBS during 4 hours, 24 hours, and 3 and 7 days. After these chase periods, cells were either fixed for confocal/EM studies or trypsinized for flow cytometry assays.

\section{Cell Treatments}

After the 4 hours POS pulse, cells were treated during the 3-day chase period with the following compounds: bafilomycin A1 (5-10 nM; Sigma-Aldrich, St. Louis, MO), Rab7 inhibitor (CID 1067700; 5-25 $\mu$ M; Sigma-Aldrich), leupeptin (10 or $25 \mu \mathrm{M}$; Calbiochem, San Diego, CA) plus Pepstatin A (25 $\mu \mathrm{M}$; Sigma-Aldrich) or a BACE 1 inhibitor (PF9283; 3-9 $\mu \mathrm{M}$; Sigma-Aldrich). After the chase period, the cells were trypsinized for flow cytometry assays.

\section{CRISPR/Cas9 Genome Engineering}

To generate CTSD knock-out (KO) ARPE-19 cells, single guide RNAs were designed for specific target sequences, as previously described. ${ }^{27}$ During single guide RNA cloning, the pSpCas9(BB)-2A-GFP (pX458) was used (a gift from Feng Zhang, Addgene plasmid \#48138, http:// n2t.net/addgene:48138, RRID: Addgene_48138). CTSD was targeted using the gRNA: ACGTTGTTGACGGAGATGCG. ARPE-19 cells were then transfected using lipofectamine 3000 , according to the manufacturer's instructions. Single colonies were grown and expanded for genomic DNA extraction. Indel mutations were confirmed by sequencing, using the primers: forward- 5'- TTTCTCTGTGCTGCCGCTTA - $3^{\prime}$, reverse- 5' - CATCGCAGCCAAGTTCGATG $3^{\prime}$. Genotyping revealed homozygous frameshift insertion (g.616_617insA).

\section{Flow Cytometry}

For flow cytometry assays, cells were trypsinized with TrypLE Express Enzyme (Gibco) for 30 minutes. Cells were resuspended in DMEM supplemented with 10\% FBS, washed two times with PBS and one time with flow cytometry buffer (1\% FBS and $2 \mathrm{mM}$ EDTA in PBS). Finally, cells were resuspended in flow cytometry buffer and data acquisition was performed in a FACS CANTO II flow cytometer (BD Biosiences) using the $488 \mathrm{~nm}$ excitation wavelength to evaluate cellular AF. At least 30,000 cells were acquired per condition using BD FACSDiva software (Version 6.1.3, BD Biosciences). Data analysis was performed in FlowJo (Version 10, BD Biosciences) and GraphPad Prism (Version 7). Results were represented as the percentage of 488positive cells and were normalized to the values of $\mathrm{AF}$ detected in cells without POS pulse or cells pulsed with POS but in the absence of compounds.

\section{Confocal Immunofluorescence Microscopy}

Cells grown on coverslips were fixed for 15 minutes in 4\% paraformaldehyde (Alfa Aesar, Haverhill, MA) or $100 \%$ methanol, at room temperature or $-20{ }^{\circ} \mathrm{C}$, respectively. Cells were blocked and permeabilized for 30 minutes in PBS containing $1 \% \mathrm{BSA}$ and $0.05 \%$ saponin or $0.1 \%$ TX100, according to the antibodies used. Cells were then incubated with primary antibodies, namely, mouse anti-RetP1 (ThermoFisher, MA5-11741) (work dilution [WD] of 1:100), mouse anti-LAMP1 conjugated with Alexa fluor 647 (BioLegend [San Diego, CA], clone H4A3, 328609) (WD of 1:500), rabbit anti-CTSD (Abcam, Cambridge, UK; ab75852) (WD of 1:200), rabbit anti-ZO-1 (Invitrogen; Waltham, MA; clone ZMD.437 40-2300) (WD of 1:100) or Phalloidin 488 (ThermoFisher, A12380) (WD of 1:400) for 1 hour, followed by incubation with Alexa-conjugated secondary antibodies donkey antimouse or anti-rabbit 647 (Invitrogen) (WD of 1:1000). Cell nuclei were labelled with DAPI (Sigma) $(1 \mu \mathrm{g} / \mathrm{mL})$ and cells were mounted in Mowiol mounting media (Calbiochem). AF was visualized in the $405 \mathrm{~nm}$ excitation wavelength. Images were acquired in a Zeiss LSM 710 confocal microscope or in a Zeiss LSM980 airyscan confocal in the Multiplex SR$4 \mathrm{Y}$ imaging mode, with a Plan-Apochromat $63 \times 1.4$ NA oilimmersion objective. Digital images were analyzed using LSM Image software or ImageJ. AFGs and RetP1-positive POS were quantified using ImageJ software (https://imagej. nih.gov/ij/). Ten random images of each time point were acquired using a $63 \mathrm{x}$ magnification, with zoom 1. Alexafluor 488 phalloidin was used to visualize cell limits and a Z-projection of all the slices containing cellular staining was done. The same threshold levels were applied to all images. AFGs were visualized in the $405-\mathrm{nm}$ excitation wavelength and counted using the 'Cell counter' plugin in ImageJ. Intracellular RetP1-positive POS labelled with an Alexa Fluor 647 were visualized and counted using the 633-nm excitation wavelength, to minimize interference from the $\mathrm{AF}$ signal because in this wavelength there is less AF signal. RetP1-positive aggregates with large dimensions and found attached to the outside of the cells were excluded from the counting. Results are represented as the average of the number of AFGs detected per field of view \pm SEM. The percentage of intracellular AFGs surrounded by LAMP1 or CTSD was calculated as the number of AFGs surrounded by those markers/number of total intracellular AFGs observed per field of view \pm SEM.

\section{EM}

For correlative light and EM (CLEM) and transmission EM, cells were seeded on laminin-coated photoetched gridded coverslips (MatTek Corporation, Ashland, MA) and at the indicated times of POS chase, cells were fixed with 4\% PFA (TAAB Laboratory Equipment Ltd, Berks, UK) in PBS for 30 minutes before image acquisition using an inverted Zeiss LSM710 (63x lens, NA 1.3, oil immersion). Samples were subsequently washed with PBS, fixed with 2\% PFA, 2\% glutaraldehyde (TAAB Laboratory Equipment Ltd) in $0.1 \mathrm{M}$ sodium cacodylate for 30 minutes, osmicated, and further processed for resin embedding. ${ }^{28}$ Resin blocks were sectioned (Leica Microsystems, Wetzlar, Germany; UC7) and 70-nm ultrathin serial sections collected on formvarcoated slot grids were stained with uranyl acetate and lead citrate and observed with a transmission electron microscope, Tecnai G2 Spirit (FEI) or observed with a JEOL 1010 or a JEOL 1400 Plus transmission electron microscope and imaged with an Orius SC1000B charge-coupled device camera with Digital Microgaph software (both Gatan). EM and light microscopy data sets were registered manually in Fiji (ImageJ) and Power Point (Microsoft Office 365 ProPlus, Microsoft Corp, Redmond, WA) using differential interference contrast images and serial EM images, where nuclear 
and plasma membrane features, together with lipid droplets were used as unbiased fiducials.

\section{Western Blot}

Cells were lysed in ice cold cell lysis buffer (Cell Signaling Technology, Danvers, MA) supplemented with protease and phosphatase inhibitor cocktails (Roche, Basel, Switzerland) according to the manufacturer's instructions. Lysates were pelleted for 15 minutes at $13,000 \times g$ at $4{ }^{\circ} \mathrm{C}$ and supernatants kept for protein quantification (BCA Protein Assay Kit, Thermo Scientific). Equal amounts of cellular proteins were resolved on $10 \%$ or $12 \%$ sodium dodecyl sulfate-polyacrylamide gels and subsequently transferred to nitrocellulose membranes (Bio-Rad Laboratories, Hercules, CA). Membranes were blocked using 5\% nonfat dry milk or 5\% BSA (Sigma-Aldrich) for phosphorylated proteins immunoblots in Tris-buffered saline (TBS) (50 mM Tris, 150 $\mathrm{mM} \mathrm{NaCl}, \mathrm{pH}=7.6$ ) containing $0.1 \%$ Tween-20 (SigmaAldrich) (TBS-T) for 1 hour and primary antibodies were then added in blocking solution. The following antibodies were used: mouse anti rhodopsin 1D4 (Abcam, ab5417) (WD of 1:1000) and mouse anti- $\beta$-Actin (Sigma-Aldrich, A3854) (WD of 1:25000). Primary antibody incubations were carried out at $4{ }^{\circ} \mathrm{C}$ overnight. After washing with TBS-T, the appropriate horseradish peroxidase-conjugated secondary antibody was added (WD of 1:5000 in blocking buffer) for 2 hours at room temperature. Antibody binding was detected using chemiluminescence ECL Prime Western Blotting Substrate (GE Healthcare, Chicago, IL) and imaged using a Chemidoc XRS (Bio-Rad).

\section{High Content Imaging}

For high-content confocal imaging, cells were cultured on 96-well skirtless CellCarrier plates (PerkinElmer, Norwalk, CT), and fixed using 4\% PFA in PBS. Each experimental condition was repeated in four wells. Using an Opera Phenix high-content screening system (PerkinElmer), the same three randomly selected regions were imaged in each well, and eight confocal slices separated by $1 \mu \mathrm{m}$ were acquired. For a semiautomated analysis of the high volume of images acquired by high-content imaging, macros were designed in ImageJ to batch process all images identically. For quantitating the number of DAPI-stained or AF punctae, z-stacks were combined into a maximum intensity projection, before identifying the individual objects using the 'Find Maxima' tool. The appropriate tolerance value for the 'Find Maxima' tool was applied to all images for a single staining and was predetermined subjectively using a sample of randomly selected images.

\section{Statistical Analysis}

Statistical analysis was performed using GraphPad Prism, version 7 Software. All our results are shown as mean \pm SEM. Normal distribution of data was assessed using the ShapiroWilk normality test. Data that followed a normal distribution were analyzed using one-way ANOVA followed by multiple comparisons Bonferroni or Dunnet post hoc correction, as appropriate. Data that did not follow a normal distribution were analyzed using the nonparametric Mann-Whitney $t$ test or one-way ANOVA Kruskal Wallis test, as appropriate. A $P$ value of less than .05 was considered statistically significant.

\section{Results}

\section{One Single Pulse of POS Leads to the Formation of AFGs}

To model the appearance of AF in the RPE and study its role in disease, we optimized an in vitro model whereby primary hfRPE cells grown for 3 weeks were challenged with a single pulse of purified porcine POS (Supplementary Fig. S1A) at two different concentrations, namely, 50 and $200 \mu \mathrm{g} / \mathrm{mL}$. Although most previous reports studying phagosome processing use POS labelled with a fluorescent probe and follow its fluorescence, we use unlabeled POS and follow the appearance of AF.

We used flow cytometry to quantify AF levels as described previously. ${ }^{29,30}$ Immediately after POS pulse ( 0 hour), no AF is detected, comparable with the cells in the absence of POS (Fig. 1A). AF starts to be detected 24 hours after POS pulse and progressively increases with time, at least until 7 days later. Moreover, cells fed with lower POS concentrations $(50 \mu \mathrm{g} / \mathrm{mL})$ showed lower AF levels, suggesting that AF depends on the POS concentration used. Similar results were obtained when ARPE-19 monolayers (Supplementary Fig. S1B) or primary pRPE (Supplementary Fig. S2A-C) were treated with a single pulse of POS and AF quantified by flow cytometry and high content screening, respectively.

Consistent with these results, in hfRPE AFGs were only first observed by confocal microscopy 24 hours after POS pulse and were still detected 3 and 7 days later (Fig. 1B), showing that they are very stable and not easily degraded by these cells. Quantification of the number of AFGs observed per field of view revealed a very similar pattern to that obtained using flow cytometry (Fig. 1C), showing that both techniques reliably measure the amount of AFGs in cells.

To confirm that these AFGs were indeed originating from undigested phagocytosed POS, POS internalization was assessed using an antibody that specifically recognizes the $N$-terminal (intradiscal) domain of rhodopsin (RetP1), ${ }^{31}$ the main protein component (>90\%) of the bilayer disk membranes of rod photoreceptors. As expected, immediately after the POS pulse ( 0 hour), rhodopsin-positive structures were detected dispersed throughout the cytoplasm, indicating that POS were efficiently phagocytosed; no AF was detected. Intracellular rhodopsin-positive staining decreased shortly after POS pulse ( 4 hours) and continued to decrease over time (Figs. 1B, D). Degradation of rhodopsin was also assessed by Western blot, using an antibody raised against the C-terminal (1D4 epitope) domain, ${ }^{31}$ because this domain is degraded in the phagocytic pathway before lysosomal fusion unlike the RetP1 epitope, which is only lost after phagolysosome fusion. ${ }^{31} \mathrm{~A}$ band of the expected size was detected after POS pulse ( 0 hour) and was still detected 4 hours later (Fig. 1E) but not after 24 hours, a time where RetP1 labeling is still detected (Figs. 1B, C). These data suggest that the early stages of phagosome processing are complete within 24 hours, but lysosome fusion with phagosomes remains incomplete 24 hours after the pulse of POS.

We note that large rhodopsin-positive POS aggregates, labelled with RetP1 antibody, were found attached to the apical cell membrane 24 hours after POS pulse and were still detected after 7 days (Fig. 1B; Supplementary Fig. S1C). We hypothesize that these rhodopsin-positive POS aggregates did not enter the cells and remained strongly attached to the plasma membrane, despite extensive washes. Importantly, in most imaging experiments we also stained the RPE layer 
A

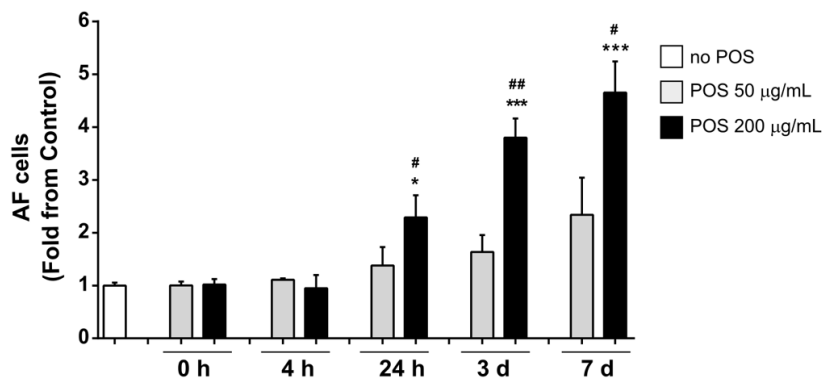

B
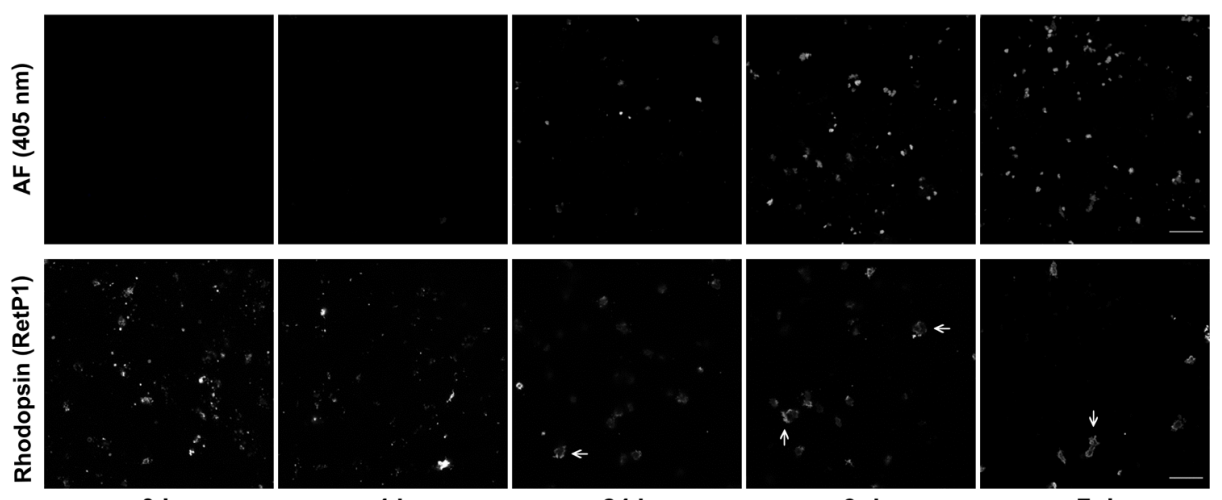

C

D
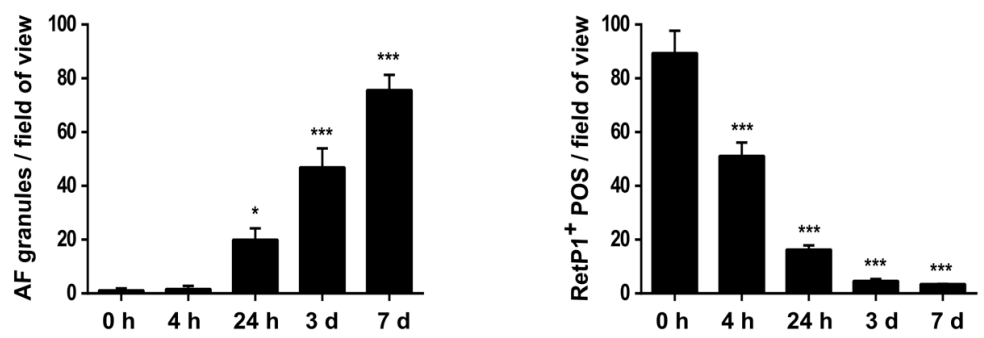

$\mathbf{E}$

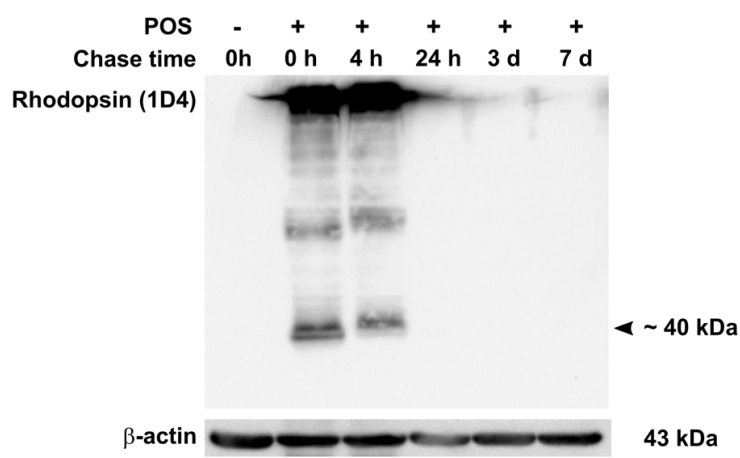

Figure 1. Lipofuscin-like AF formation in hfRPE cells. hfRPE cells pulsed with different concentrations of POS were monitored at the indicated time points after POS incubation. (A) AF levels were quantified by flow cytometry as in the Material and Methods and are represented as mean \pm SEM of at least three independent experiments, performed in duplicate. Statistical differences relative to cells with no POS were assessed using one-way ANOVA followed by Kruskal Wallis test (**** $\left.P<0.001,{ }^{*} P<0.05\right)$, and differences between cells treated with 50 and $200 \mu \mathrm{g} / \mathrm{mL}$ POS at each time point were evaluated using the Mann-Whitney $t$ test $(\# \# P<0.01 ; \# P<0.05)$. (B) AFGs were visualized by confocal immunofluorescence microscopy. Internalized rhodopsin-positive POS were detected using an anti-rhodopsin antibody (RetP1). Phalloidin staining was used to monitor cell limits and a Z-projection is represented. Arrows point to RetP1-positive POS aggregates that are attached to the apical cell membrane. Scale bar: $20 \mu \mathrm{m}$. (C) The number of intracellular AFGs were quantified using ImageJ and are represented as number of AFGs per field of view \pm SEM of three independent experiments, where at least 10 random images were analyzed per assay. (D) Intracellular RetP1-positive POS were quantified using ImageJ and are represented as number of RetP1-positive POS per field of view \pm SEM of 3 independent experiments, where at least 10 random fields were analyzed per assay. Statistical comparison was performed using one-way ANOVA followed by Dunnet's or Bonferroni's multiple comparison test $\left({ }^{*} * * * P<0.001\right.$, ${ }^{*} P<0.05$ ). (E) POS degradation was assessed using an anti-rhodopsin antibody (1D4) by Western blot analysis. Images are representative of two independent experiments. 
with phalloidin and analyzed only granules that were clearly within the cytoplasm.

Our results show that one single pulse of human fetal primary RPE cells with porcine POS leads to the formation of stable AFGs, mimicking the appearance of lipofuscin in vivo. Moreover, AFG appearance is dependent on POS concentration and increases with time. These data confirm that AF derives from POS and suggests that lysosome fusion is required for $\mathrm{AF}$ appearance.

\section{Intracellular AFGs Contain Undigested POS, Are Bound by a Single Membrane, and Contain Late Endosome/Lysosome Markers}

To confirm that AFGs result from phagolysosome formation, we performed CLEM and studied AFGs 3 days after the POS pulse (Fig. 2A). At this time point, AFGs seem to be contained within a continuous single membrane and contain within their lumen membrane structures that resemble a more disorganized version of the regular shaped isolated segment membranes characteristic of in retina POS $^{31}$ and the isolated purified fractions of our porcine POS preparations (Supplementary Fig. S1A). Very similar membranebound content was found in samples at 7 days after POS pulse (Fig. 2B).

These results predict that AFGs contain markers of phagolysosomes. ${ }^{32,33}$ Therefore, we immunostained RPE cells with the established lysosome markers, LAMP1 and CTSD (Figs. 3A, B). At 3 and 7 days after the POS pulse, approximately $70 \%$ of the intracellular AFGs were observed to be at least partially surrounded by the lysosomal membrane marker LAMP1 (Figs. 3A, C). A smaller percentage of AFGs, about $20 \%$ exhibit staining for the luminal hydrolase CTSD, was observed surrounding the AF granules (Figs. 3B, D).

We conclude that AFGs are stable over time, contain the remains of ingested POS within a single membrane, and contain lysosome markers, consistent with granules that evolve from phagolysosomes.

\section{Lysosome Fusion Is Required for AFG Formation, But Lysosome Dysfunction Enhances AF Accumulation}

We next explored the role of lysosomes in AFG formation. We started by interfering with phagosome-lysosome fusion using a Rab7 inhibitor, CID1067700. ${ }^{34}$ Rab7 is a Ras-like GTPase that is crucial for endo-lysosome and phago-lysosome fusion (reviewed $\mathrm{in}^{35}$ ). Incubation with CID1067700 for 3 days significantly decreased AF levels, in a dose-dependent manner, when compared with control cells in the absence of the inhibitor (Fig. 4A).

The formation of AFGs after POS phagocytosis indicates that phagolysosome formation did not always lead to complete digestion, at least in a proportion of them. The AFGs seem to contain partially digested POS by CLEM and transmission EM (Fig. 2), as discussed elsewhere in this article, and the AF probably results from lipid oxidation within the phagolysosome lumen. We hypothesized that the resultant AFGs are dysfunctional phagolysosomes. Therefore, we attempted to disrupt lysosomal function and observe its effects. We first fed RPE cells with POS and later treated with the compounds indicated to ensure that drug treatment did not interfere with POS binding or internalization.
We started by interfering with lysosomal $\mathrm{pH}$ using bafilomycin A1. Bafilomycin A inhibits the lysosomal proton pump V-ATPase, and thus impairs lysosome acidification and indirectly lysosomal enzyme activity. ${ }^{36}$ We observed that increasing concentrations of bafilomycin A led to increasing levels of AF appearance (Fig. 4B). This finding suggests that, in the presence of a high number of fusogenic lysosomes, an acidic lysosomal $\mathrm{pH}$ is critical for a complete POS digestion, and that AFGs result from incompletely digested POS.

We then interfered with hydrolytic activity of lysosomes. First, we evaluated AF formation in the presence of the protease inhibitors leupeptin and pepstatin A, powerful inhibitors of lysosomal enzymes. Cells incubated with increasing concentrations of these inhibitors showed increasing AF levels in RPE cells (Fig. 4C). Similar results were obtained when cells were incubated with the BACE1 inhibitor PF-7802 (Fig. 4D), described as having specific CTSD inhibitor activity as an off-target effect. ${ }^{37}$

As an alternative to using drugs, which can induce undesired off-target effects, we generated a CTSD knockout (KO) ARPE-19 (19-CTSD-KO) cell line using CRISPR/Cas9 (Supplementary Fig. S3A), to induce lysosome dysfunction. CTSD has been described as the primary lysosomal enzyme involved in POS degradation in RPE cells. ${ }^{30,38,39}$ As expected, 19-CTSD-KO no longer express the pro-form or the mature form of the CTSD protein (Supplementary Fig. S3B). AFGs were detected 24 hours after POS pulse in wild-type (WT) and 19-CTSD-KO cells (Fig. 4E), phenocopying the results obtained in hfRPE cells shown in Figure 1. Quantification of the number of AFGs observed by microscopy showed that 19-CTSD-KO cells contain more AFGs in all the time points studied when compared with WT cells (Fig. 4E and Supplementary Fig. S3C). The same trend was observed using flow cytometry (Fig. 4F). Interestingly, 19-CTSD-KO cells have higher basal levels of AF (at the 488-nm wavelength) in the absence of POS when compared with WT cells (Fig. 4F), possibly because cargo degradation is chronically impaired in these cells, thus generating AF. Nevertheless, basal AF detected in these cells is clearly distinct from POS-derived AF because intracellular AF granules are exclusively detected in cells pulsed with POS (Fig. 4E).

Unlike hfRPE, adult primary porcine pRPE monolayers contain a lysosomal system that was previously operating within an intact retina and therefore presumably contain fully operational lysosomes. Nevertheless, these cells also produce AFGs after treatment with a single pulse of POS in a time-dependent manner (Supplementary Fig. S2A, B). Because these are adult cells, some AFGs are present before the POS pulse, presumably acquired during the lifetime of the animal from which they were derived. To distinguish between POS-derived AFGs and previously developed AFGs, we loaded the POS with gold (Supplementary Fig. S4) and performed CLEM so that AFGs containing POSderived gold could be identified. Some intense AFGs seemed to lack gold particles, so they may have been preformed, although we cannot exclude the presence of gold particles in another section plane (Fig. 5A). In addition, less intense AFGs contained POS-derived gold, in agreement with the data from hfRPE, indicating that the AFGs are POS derived. The gold particles in the AFGs are clearly aggregated, indicating that the POS are at least partly broken down, liberating the gold particles that they contain, which have aggregated in the acidic lumen of the lysosome (Fig. 5A).

Our data with hfRPE indicated that, although delivery of POS to lysosomes is necessary for AF production, 
A
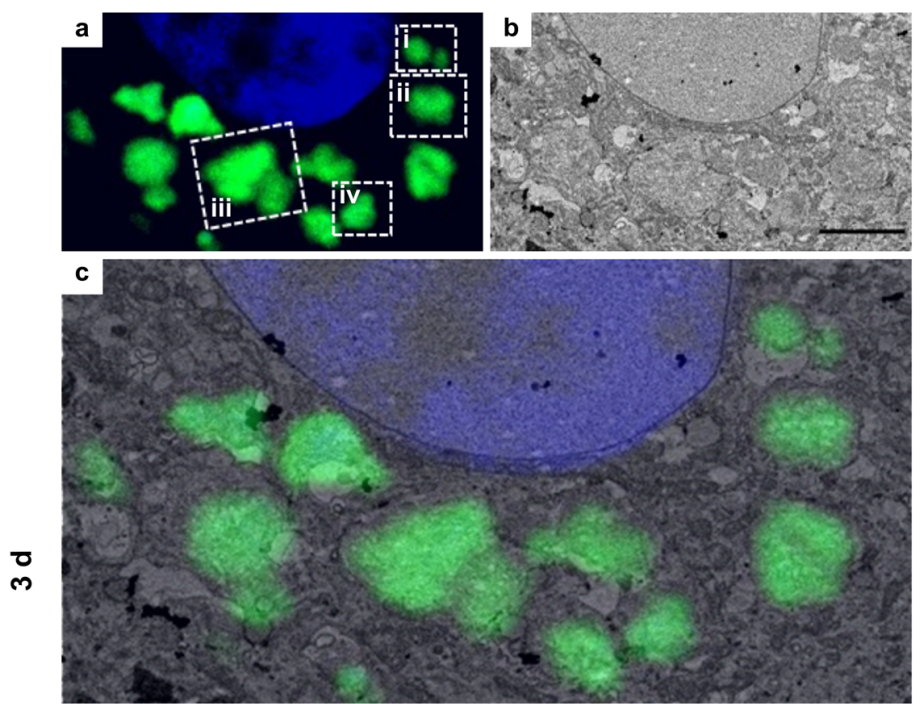
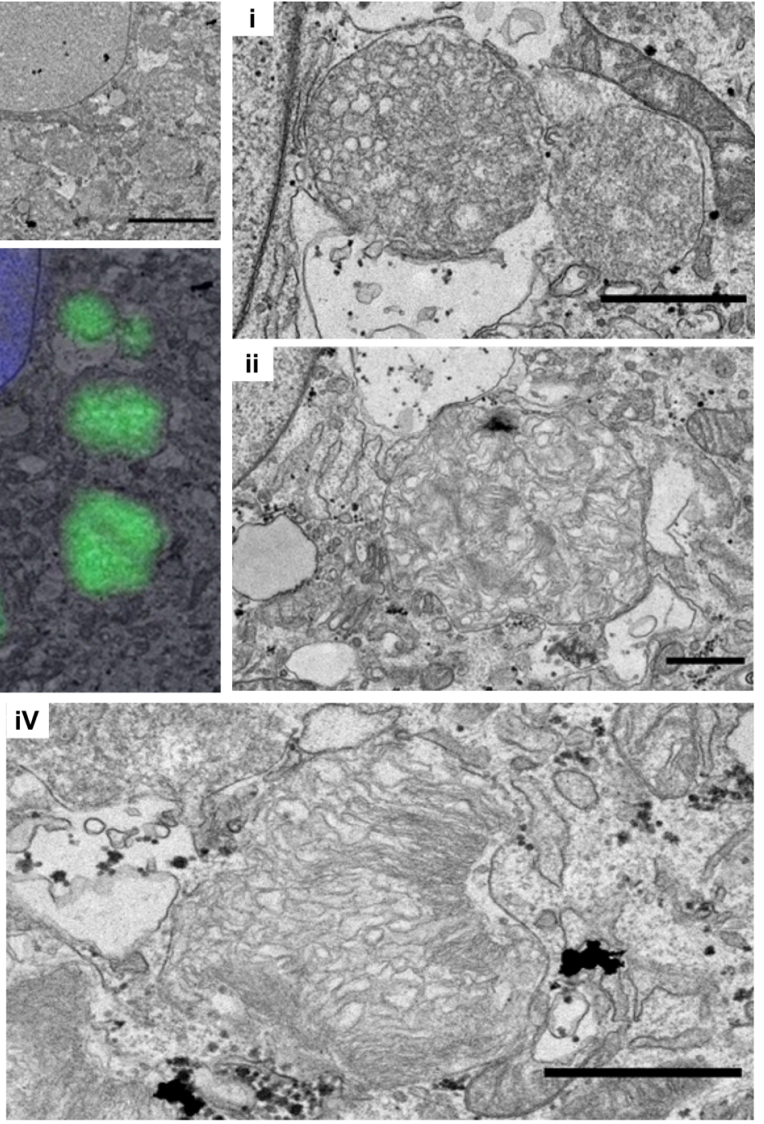
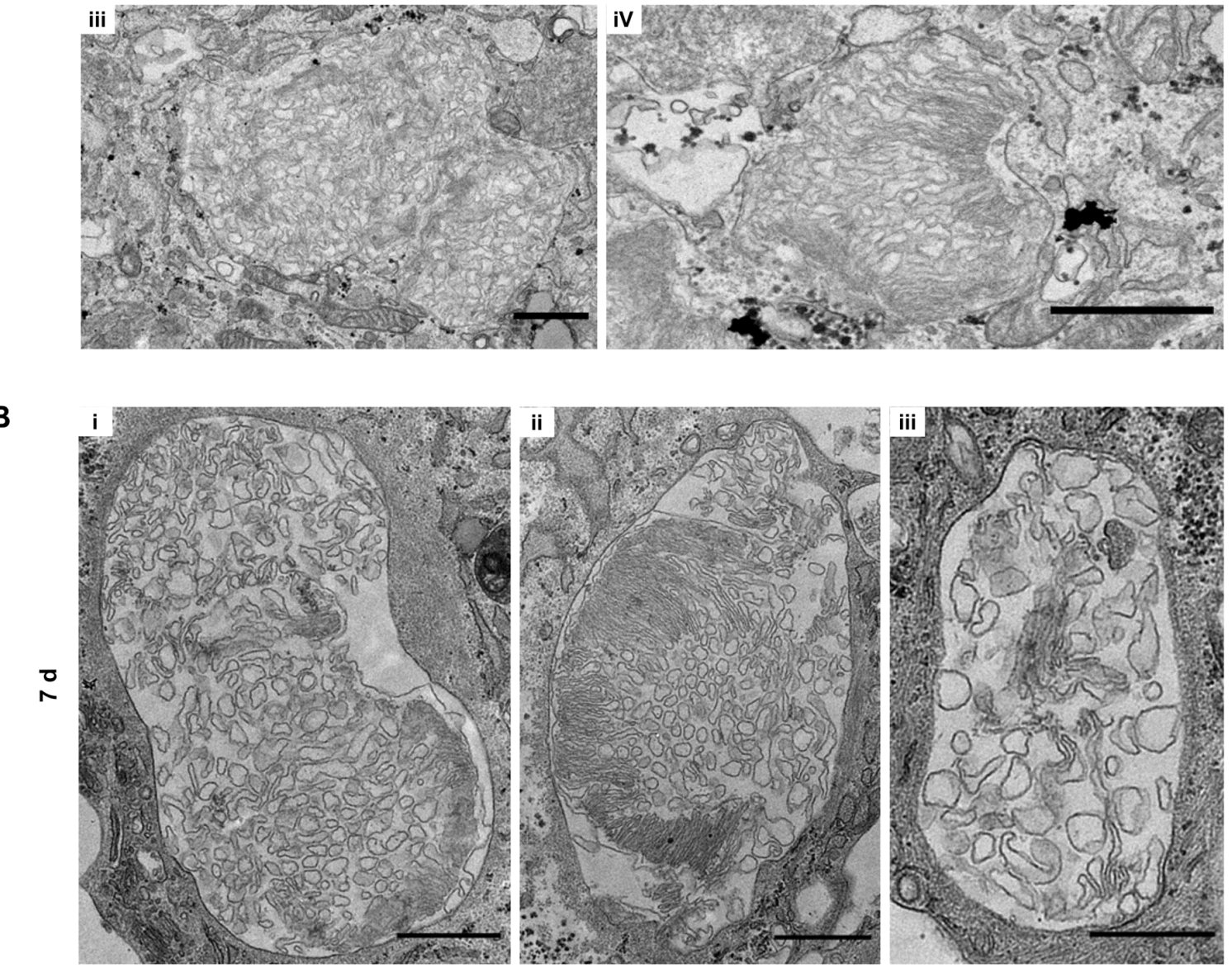

Figure 2. AFGs are surrounded by a single membrane and contain POS-like material. (A) hfRPE grown on Matek gridded dishes were fed with POS, washed, and chased for 3 days. Samples were processed for CLEM as in the Materials and Methods. (a) Confocal stack showing nucleus in blue and AFGs in green. (b) Transmission EM section of the equivalent area of the same cell as in a. Scale bar: $5 \mu \mathrm{m}$. (c) overlay of a and b. (i-iv). Transmission EM insets of areas shown in a. Scale bars: $1 \mu \mathrm{m}$. (B) hfRPE grown on coverslips were processed for transmission EM 7 days after POS pulse. Examples of membrane-bound AFGs. Scale bars: i and ii, $1 \mu \mathrm{m} ;$ iii, $0.5 \mu \mathrm{m}$. Three independent samples were prepared for each time point and more than five AFGs were analyzed in each sample.

lysosome dysfunction and hence incomplete degradation of POS, increased AF production. However, we found that the pRPE have limited tolerance to inhibitors of lysosome function. Instead, we mildly UV-irradiated POS before feeding to the cells to inhibit POS degradation. Treatment of RPE cells with UV-irradiated POS has previously been shown to induce accumulation of $\mathrm{AF}^{25,33,40}$ and we found that although UV POS pretreatment had no clear effect in hfRPE, it clearly elevated AF production in adult pRPE (Supplementary Fig. S2D). CLEM of pRPE treated with gold loaded 
A

ర
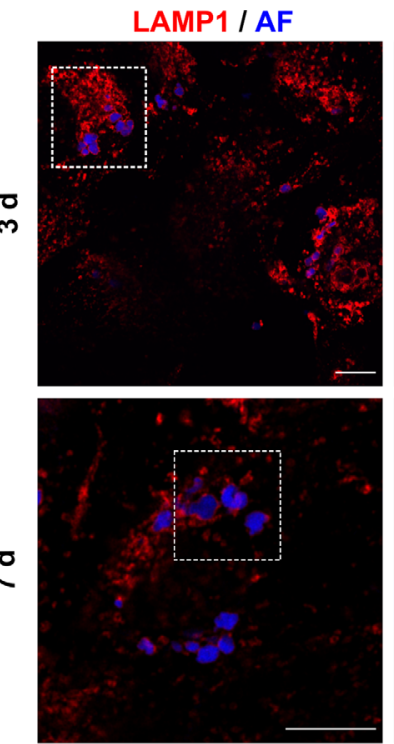

B

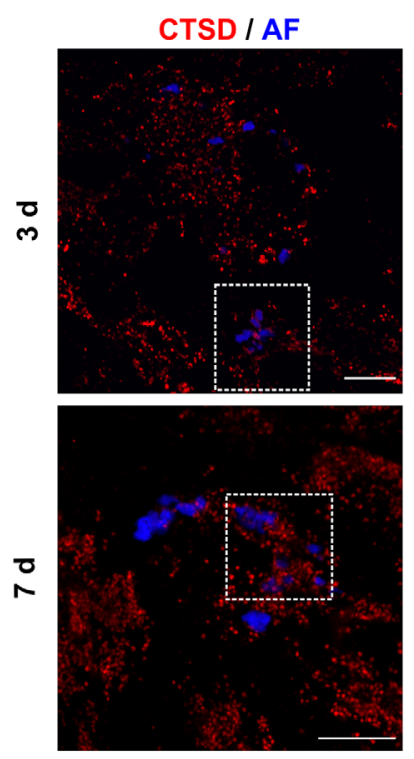

C

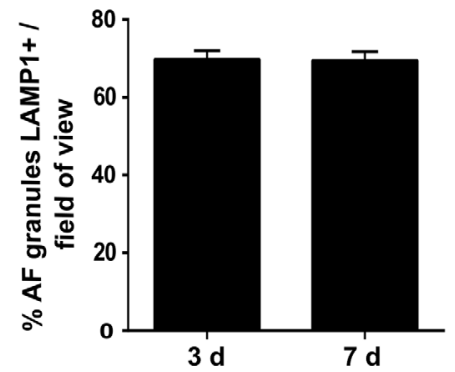

ZOOM
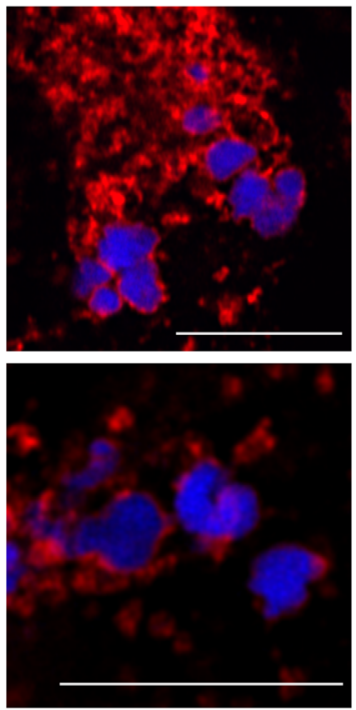

ZOOM
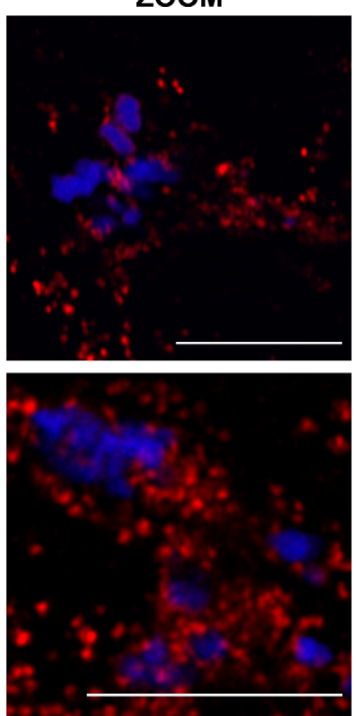

D

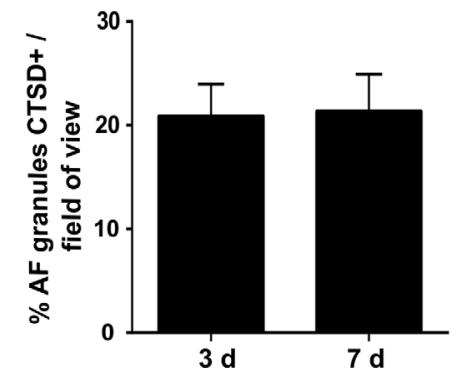

AF
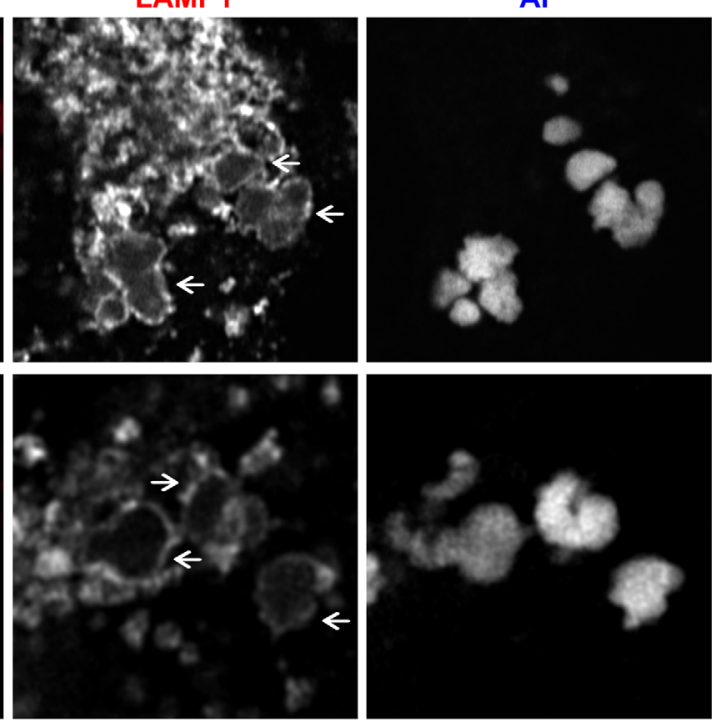

CTSD
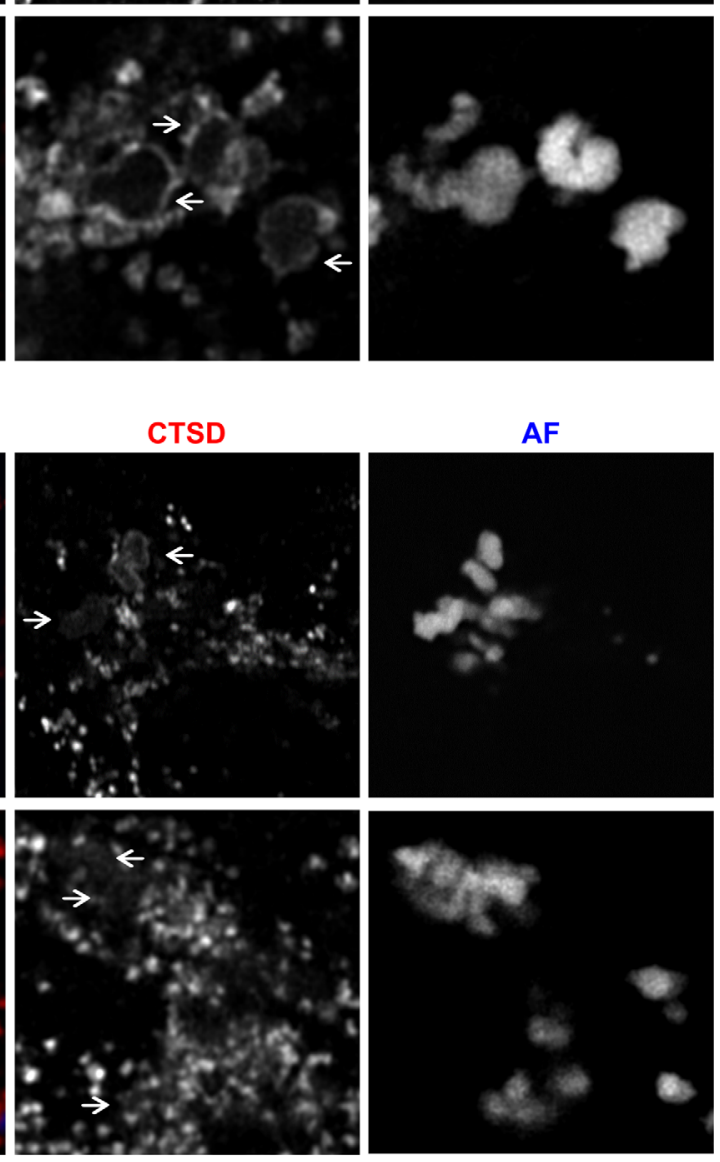

$\mathrm{AF}$

Figure 3. AFGs colocalize with late endosome/lysosome markers. hfRPE pulsed with $200 \mu \mathrm{g} / \mathrm{mL}$ POS and chased for the indicated time points were stained with (A) mouse anti-LAMP1 (red) or (B) rabbit anti-CTSD antibodies (red) and AF (blue). Only a Z-slice is represented. The region outlined with a square was zoomed (ZOOM) and the different channels separated. Arrows indicate areas where AFGs are contained/surrounded by LAMP1 or CTSD staining. Scale bar: $10 \mu \mathrm{m}$. AFGs surrounded by LAMP1 (C) or CTSD (D) were quantified using Image and are represented as the percent of granules per field of view \pm SEM of three to five independent experiments, where at least 10 cells were analyzed per assay. 
A. Rab7 inhibitor

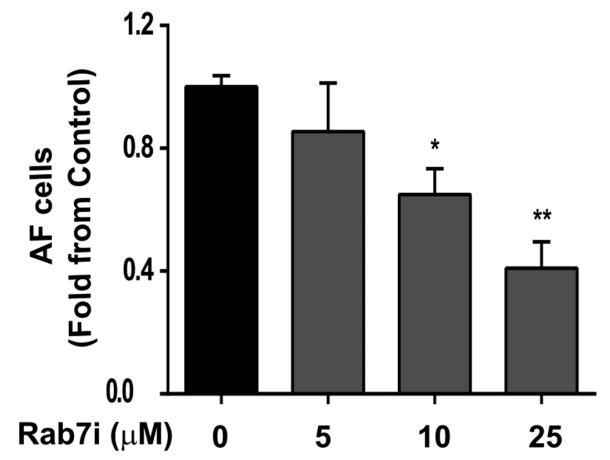

C. Leupeptin/ Pepstatin A

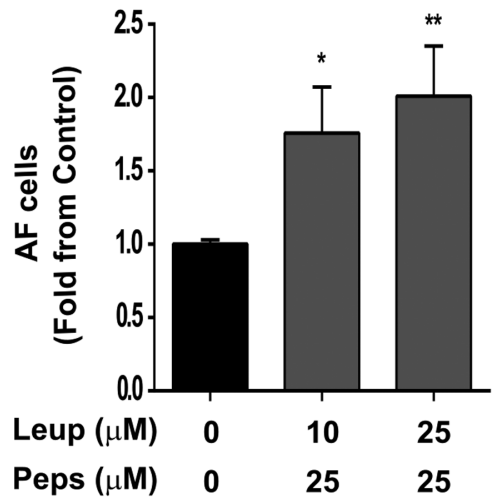

E. Cathepsin D KO

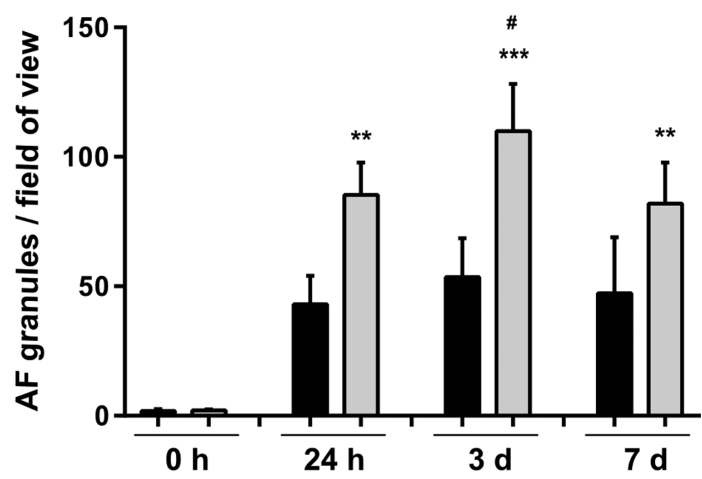

B. Bafilomycin A1

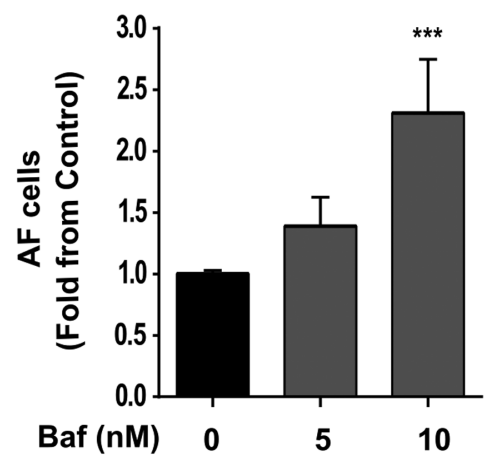

D. BACE inhibitor

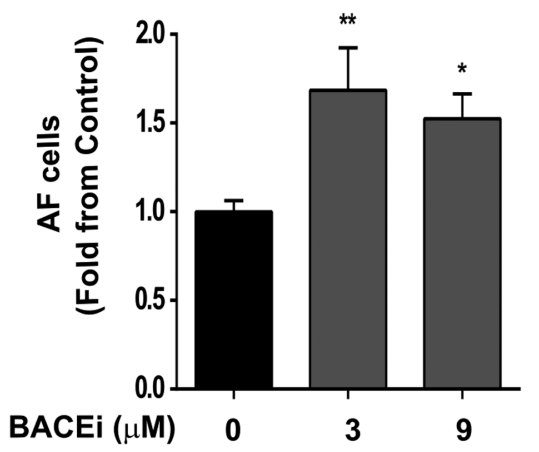

F. Cathepsin D KO

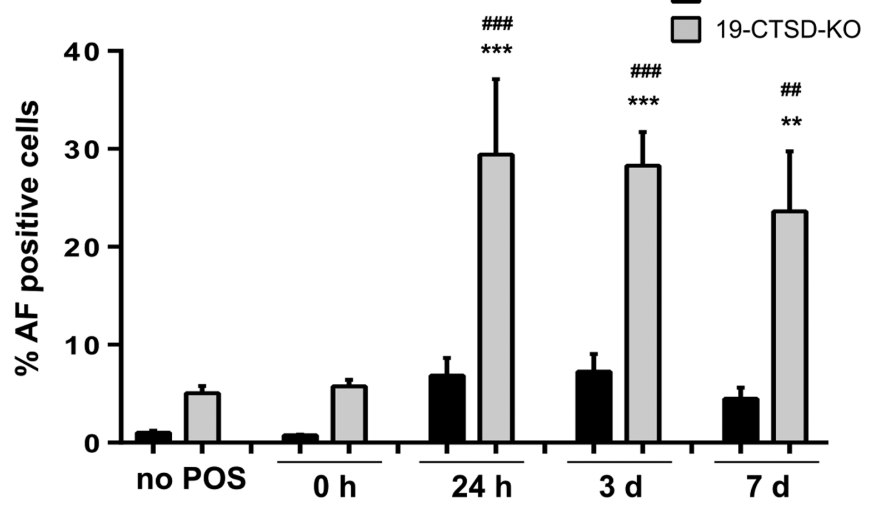

Figure 4. LYSOSOME FUNCTION AFFECTS AFG FORMATION. hfRPE were pulsed with $200 \mu \mathrm{g} / \mathrm{mL}$ POS and incubated for 3 days with different concentrations of (A) Rab7 inhibitor, (B) bafilomycin A1, (C) leupeptin and pepstatin A, and (D) BACE inhibitor. AF levels were quantified by flow cytometry as in the Material and Methods. Results are represented as mean \pm SEM of at least three independent experiments, performed in duplicate. Statistical comparison was performed using one-way ANOVA followed by Dunnet's or Bonferroni's multiple comparison test or the Kruskal-Wallis test, in the case of Rab7 inhibitor $\left(* * * P<0.001,{ }^{* * *} P<0.01,{ }^{*} P<0.05\right.$ ). (E) ARPE-19 wild type (WT) and 19-CTSD-KO cells were fed with POS and chased for different time periods. Intracellular AFGs visualized by confocal immunofluorescence microscopy were quantified using ImageJ and are represented as number of AFGs per field of view \pm SEM of three independent experiments, where at least 10 random fields were analyzed per assay. (F) ARPE-19 WT and 19-CTSD-KO AF-positive cells were quantified by flow cytometry as in the Material and Methods at different time points after POS pulse. Results are represented as mean \pm SEM of four independent experiments, performed in duplicate. Statistical comparison was performed using one-way ANOVA followed by Dunnet's or Bonferroni's multiple comparison test, significant differences are relative to no POS (*** $P<0.001$; *** $P<0.01$ ) or between ARPE-19 and 19-CTSD-KO cells at each time point $\left({ }^{\# \#} P<0.001,{ }^{\# \#} P<0.01 ;{ }^{\#} P<0.05\right)$. 

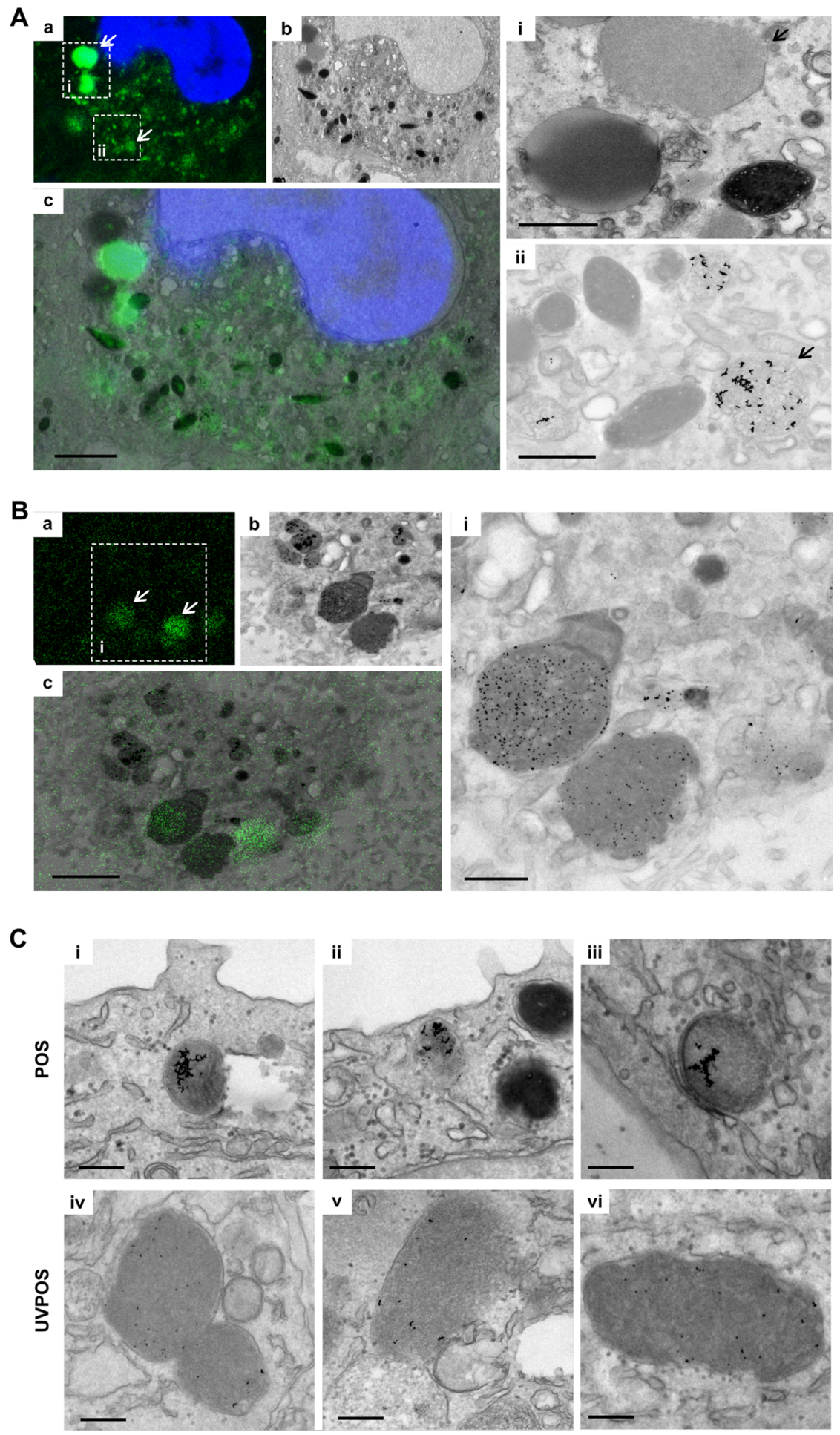

FigurE 5. AFGs contain undigested gold-labelled POS in pRPE. Representative images of pRPE grown on Matek gridded dishes pulsed with (A) gold-tagged POS or (B) UV-irradiated POS (UVPOS), washed and chased for 3 days. Samples were processed for CLEM as in the Materials and Methods. (a) Confocal stack showing nucleus in blue and AFGs in green. (b) Transmission EM section of the equivalent area of the same cell as in (a). (c) Overlay of (a) and (b). Scale bar: $2.5 \mu \mathrm{m}$. (i and ii) Transmission EM insets of areas shown in (a). Scale bars: 1 $\mu \mathrm{m}$. (C) pRPE grown on coverslips were processed for transmission EM 5 days after pulse with gold-tagged POS or UVPOS. The presence of gold in persistent AFGs confirms that they are derived from POS. Scale bar: $500 \mathrm{~nm}$.

UV-irradiated POS showed that intense AFGs contained nonaggregated gold particles, indicating incomplete degradation of POS and/or elevation of lysosomal pH (Fig. 5B). A comparison of cells treated with a single pulse of POS or
UV POS and chased for 5 days clearly shows that UV POSderived gold accumulates in larger granules where it remains monodisperse, compared with the smaller granules containing aggregated POS-derived gold (Fig. 5C). Thus, using a 
different approach to targeting the lysosome directly, we have provided further evidence that incomplete POS degradation enhances AFG formation.

\section{Discussion}

The present results confirm previous studies suggesting that AFG derive from phagocytosed POS and further suggest that some lysosome activity, in particular phagosome-lysosome fusion, is required for AFGs formation. However, lysosome dysfunction, particularly a loss of CTSD activity, leading to the incomplete digestion of POS is required for the appearance of AF and the formation of stable AFGs. Therefore, decreased lysosomal activity leads to less degradative capacity, decreased POS degradation, and increased AFGs accumulation.

Previous studies have reported that repeated phagocytosis of POS leads to the progressive accumulation of AFGs within cultured primary RPE cells and RPE cell lines. ${ }^{19-25}$ Here we optimized a method which leads to the accumulation of AFGs after a single pulse of POS (or UV-irradiated POS), presumably owing to the high concentration of the POS material. Unlike other tissues (notably neurons), RPE AFGs have a known source and their appearance can be induced by pulsing RPE with POS, representing a dose and time-dependent assay. Because AFGs are surrogates of RPE lipofuscin, a marker of cell stress and disease, this assay allows a unique opportunity to study the mechanisms of formation of AFGs, a process that remains poorly characterized. Here we confirm previous studies that suggest that AFGs/lipofuscin derive from POS phagocytosis. ${ }^{19-25,29,30}$ An inverse correlation was observed between rhodopsin staining and AF (Fig. 1). In fact, as rhodopsin staining of phagosomes decreased, AF progressively developed. Consistently, gold particles phagocytosed within POS, and liberated as POS are degraded, are found within AFGs. AF is detected by 24 hours after POS pulse and progressively increases over 7 days (Fig. 1). POS-derived AF is clearly distinct from AF generated by autophagy of endogenous material. ARPE 19-CTSD KO cells show higher basal AF levels, but do not exhibit accumulation of intracellular AF granules in the absence of POS. AFGs similar to lipofuscin are only detected after a POS pulse. At 3 and 7 days after the pulse, the AFGs looked similar and exhibited partially digested POS surrounded by a single membrane (Fig. 2). These results suggest that AFGs have not lysed and released their content into the cytoplasm. Furthermore, a single membrane was always observed on the periphery of these granules at the time points studied. Lipofuscin studied in intact retinas are bounded similarly by a single membrane but contain a homogeneous lumen. ${ }^{14}$ This difference is presumably due to the much longer maturation time of lipofuscin compared with AFGs observed in this study. Similar single membranebound AFGs have been reported in RPE-J cells, ${ }^{41}$ suggesting that this is indeed a process of phagocytosis and not autophagy.

Having established the source of AFGs from phagosomes, we asked whether AFGs exhibited characteristics of phagolysosomes. We stained AFGs with two widely used markers of lysosomes, the lysosome membrane marker LAMP1, which clearly stained the limiting membrane of a majority of AFGs, and the luminal enzyme CTSD shown to surround AF in only around $20 \%$ of AFGs (Fig. 3). These results confirmed that AFGs have resulted from the fusion of lysosomes to form phagolysosomes. Because our EM results do not show an accumulation of lysosomes surrounding AF granules, we conclude that CTSD is consumed within AFGs, whereas LAMP1 is retained at the surface of the granules. However, we cannot completely exclude that AF granules are surrounded by lysosomes. A recent study followed the fate of POS and oxidatively modified POS in ARPE19 cells for 3 days and found that POS were sequentially trafficking to LAMP1 and LAMP2 as reported here, but in that study the use of fluorescently labelled POS prevented the analysis of formation of AFGs. ${ }^{32,33,41}$ In post mortem RPE derived from patients with AMD, enlarged and annular LAMP1-positive compartments were observed, ${ }^{42}$ which could represent AFGs, even though AF was not studied.

Several reports have also shown LC3 colocalization with internalized fluorescently labelled POS, ${ }^{32,33,41}$ as LC3associated phagocytosis contributes to efficient POS degradation and recycling of bis-retinoids. ${ }^{38}$ However, we were unable to colocalize LC3 with AFGs either formed in hfRPE or in ARPE-19 cells (results not shown). This difference could be due to technical limitations related to the weak anti-LC3 signal obtained in our cells, under our testing conditions. Alternatively, only a proportion of phagosomes are reported to stain for LC3 at a given time ${ }^{43}$; thus, AFGs may form from LC3-negative phagosomes or have lost LC3 during the maturation of AFGs.

The formation of phagolysosomes (or endolysosomes) normally leads to a complete digestion of the phagosome material, with reformation of "terminal" lysosomes. ${ }^{44}$ Although some POS-phagosomes will be fully digested, the formation of AFGs indicates that at least a proportion is not. The AFGs seem to contain partially digested POS by CLEM and transmission EM (Fig. 2), as discussed elsewhere in this article, and the AF probably results from POS degradation, suggesting that they represent dysfunctional lysosomes, as observed in many pathological conditions, from lysosome storage diseases to age-related neurodegenerative diseases. ${ }^{15}$ We show here that classical ways of inducing lysosomal dysfunction lead to increased AF in RPE cells. In fact, disruption of lysosomal $\mathrm{pH}$ (bafilomycin A) or hydrolytic activity (CTSD, leupeptin/pepstatin) promote increased intensity of AF within AFGs and/or increased numbers of AFGs. Our results are in agreement with previous reports showing CTSD as the primary lysosomal enzyme involved in POS degradation. The expression of a mutated inactive form of CTSD lead to impaired processing of POS in RPE cells ${ }^{38,39}$ and in a mouse model of CLN10 disease, the absence of CTSD increased the accumulation of storage material with age. ${ }^{45}$ Nevertheless, different cathepsins and other lysosomal enzymes, such as cysteine proteases and lipases, probably play equally important roles in POS degradation. ${ }^{4}$ This point should be addressed further in future studies.

Additionally, the prevention of full degradation of POS by mild UV irradiation of the POS also increased AF in adult pRPE. In agreement with the present studies, ammonium chloride, an agent that increase lysosomal $\mathrm{pH}$ and therefore serves as a lysosomal inhibitor, induced the appearance of $\mathrm{AF}$ in RPE even in the absence of POS and an eight-fold increase in AF after continuous POS pulse. ${ }^{46}$ Conversely, acidification of lysosomes via acidic nanoparticles and pharmacological agents reduce AF in RPE cells. ${ }^{20,29,30}$

What could be the source of AF within the newly formed granules? Although not the scope of the present study, it is likely that lipid incomplete degradation and modification may be more significant than protein as the main 
generator of $\mathrm{AF}$ as previous studies showed that incubation of hRPE cells with lipid peroxidation products, such as 4-hydroxynonenal and malondialdehyde resulted in accumulation of granular material with lipofuscin-like $\mathrm{AF}^{22,46}$ Conversely, in the present study we present conflicting data regarding the contribution of protein to AF. Although rhodopsin is degraded rapidly as observed by both Western blot and confocal microscopy, CTSD KO significantly enhanced cellular AF. Future studies should clarify this issue.

In summary, the present study establishes that lysosomal dysfunction is a major contributor in AFG formation. These observations suggest that therapies targeted towards improvement of lysosome function, such as via a decrease in the $\mathrm{pH}$ as proposed by Mitchell and colleagues ${ }^{29,30}$ or lysosome rejuvenation, such as via autophagy activation, are promising avenues to delay the progression of intermediate or dry AMD, currently an important and unmet medical need.

\section{Acknowledgments}

The authors thank the Cell Culture facility at CEDOC, the scientific and technical assistance of T. Pereira, C. Andrade, and A. Farinho from the CEDOC Flow Cytometry, Microscopy and Histology facilities; and A.L. Sousa and E.M. Tranfield at the Instituto Gulbenkian de Ciência Electron Microscopy Facility, for technical expertise and help with EM optimisation and processing. We also acknowledge our local slaughterhouse (CASOCentro de Abate de Suínos do Oeste) that generously provided porcine eyes.

Supported by Fundação para a Ciência e Tecnologia (FCT) - Portugal co-funded by FEDER under the PT2020 Partnership Agreement (to MCS, including project PTDC/MEDPAT/30385/2017, iNOVA4Health-UIDB/04462/2020, research infrastructure PPBI-POCI-01-0145-FEDER-022122, MERA.NET 2/0005/2016), Boehringer Ingelheim (to MCS), Fight for Sight UK (to MCS), Wellcome Trust grant number $212216 / \mathrm{Z} / 18 / \mathrm{Z} /$ (to CEF). MJH was funded by Moorfields Eye Charity with the Bill Brown 1989 Charitable Trust PhD studentship 538158, MLS was funded by FCT-CEECIND/01536/2018, ACF was funded by FCT PhD studentship (PD/BD/135503/2018). This work was developed with the support from the research infrastructure PPBI-POCI01-0145-FEDER-022122, co-financed by FCT (Portugal) and Lisboa2020, under the PORTUGAL2020 agreement (European Regional Development Fund) and this article is supported by the LYSOCIL project funded by the European Union's Horizon 2020 programme under grant agreement No. 811087.

Disclosure: C. Escrevente, None; A.S. Falcão, None; M.J. Hall, None; M. Lopes-da-Silva, None; P. Antas, None; M.M. Mesquita, None; I.S. Ferreira, None; M.H. Cardoso, None; D. Oliveira, None; A.C. Fradinho, None; T. Ciossek, Boehringer Ingelheim (E); P. Nicklin, Boehringer Ingelheim (E); C.E. Futter, None; S. Tenreiro, None; M.C. Seabra, None

\section{References}

1. Wong WL, Su X, Li X, et al. Global prevalence of agerelated macular degeneration and disease burden projection for 2020 and 2040: a systematic review and meta-analysis. Lancet Glob Health. 2014;2(2):e106-e116.

2. Ardeljan D, Chan CC. Aging is not a disease: distinguishing age-related macular degeneration from aging. Prog Retin Eye Res. 2013;37:68-89.
3. Bonilha VL. Age and disease-related structural changes in the retinal pigment epithelium. Clin Ophthalmol. 2008;2(2):413-424.

4. Lakkaraju A, Umapathy A, Tan LX, et al. The cell biology of the retinal pigment epithelium. Prog Retin Eye Res. 2020:100846.

5. Handa JT, Bowes Rickman C, Dick AD, et al. A systems biology approach towards understanding and treating non-neovascular age-related macular degeneration. Nat Commun. 2019;10(1):3347.

6. Bermond K, Wobbe C, Tarau IS, et al. Autofluorescent granules of the human retinal pigment epithelium: phenotypes, intracellular distribution, and age-related topography. Invest Ophthalmol Vis Sci. 2020;61(5):35.

7. Einbock W, Moessner A, Schnurrbusch UE, Holz FG, Wolf S, FAM Study Group. Changes in fundus autofluorescence in patients with age-related maculopathy. Correlation to visual function: a prospective study. Graefes Arch Clin Exp Ophthalmol. 2005;243(4):300-305.

8. Schmitz-Valckenberg S, Fleckenstein M, Scholl HP, Holz FG. Fundus autofluorescence and progression of age-related macular degeneration. Surv Ophthalmol. 2009;54(1):96-117.

9. Ueda K, Zhao J, Kim HJ, Sparrow JR. Photodegradation of retinal bisretinoids in mouse models and implications for macular degeneration. Proc Natl Acad Sci USA. 2016;113(25):6904-6909.

10. Moreno-Garcia A, Kun A, Calero O, Medina M, Calero M. An Overview of the Role of Lipofuscin in Age-Related Neurodegeneration. Front Neurosci. 2018;12:464.

11. Warburton S, Davis WE, Southwick K, et al. Proteomic and phototoxic characterization of melanolipofuscin: correlation to disease and model for its origin. Mol Vis. 2007;13:318329.

12. Feeney-Burns L, Eldred GE. The fate of the phagosome: conversion to 'age pigment' and impact in human retinal pigment epithelium. Trans Ophthalmol Soc UK. 1983;103(Pt 4):416-421.

13. Ishikawa T, Yamada E. The degradation of the photoreceptor outer segment within the pigment epithelial cell of rat retina. J Electron Microsc (Tokyo). 1970;19(1):85-99.

14. Feeney L. Lipofuscin and melanin of human retinal pigment epithelium. Fluorescence, enzyme cytochemical, and ultrastructural studies. Invest Ophthalmol Vis Sci. 1978;17(7):583-600.

15. Ballabio A, Bonifacino JS. Lysosomes as dynamic regulators of cell and organismal homeostasis. Nat Rev Mol Cell Biol. 2020;21(2):101-118.

16. Ferrington DA, Sinha D, Kaarniranta K. Defects in retinal pigment epithelial cell proteolysis and the pathology associated with age-related macular degeneration. Prog Retin Eye Res. 2016;51:69-89.

17. Carmona-Gutierrez D, Hughes AL, Madeo F, Ruckenstuhl C. The crucial impact of lysosomes in aging and longevity. Ageing Res Rev. 2016;32:2-12.

18. Lie PPY, Nixon RA. Lysosome trafficking and signaling in health and neurodegenerative diseases. Neurobiol Dis. 2019;122:94-105.

19. Boulton M, McKechnie NM, Breda J, Bayly M, Marshall J. The formation of autofluorescent granules in cultured human RPE. Invest Ophthalmol Vis Sci. 1989;30(1):82-89.

20. Guha S, Baltazar GC, Coffey EE, et al. Lysosomal alkalinization, lipid oxidation, and reduced phagosome clearance triggered by activation of the $\mathrm{P} 2 \mathrm{X} 7$ receptor. FASEB J. 2013;27(11):4500-4509.

21. Kaemmerer E, Schutt F, Krohne TU, Holz FG, Kopitz J. Effects of lipid peroxidation-related protein modifications on RPE lysosomal functions and POS phagocytosis. Invest Ophthalmol Vis Sci. 2007;48(3):1342-1347. 
22. Lei L, Tzekov R, McDowell JH, Smith WC, Tang S, Kaushal S. Formation of lipofuscin-like material in the RPE Cell by different components of rod outer segments. Exp Eye Res. 2013;112:57-67.

23. Nilsson SE, Sundelin SP, Wihlmark U, Brunk UT. Aging of cultured retinal pigment epithelial cells: oxidative reactions, lipofuscin formation and blue light damage. Doc Ophthalmol. 2003;106(1):13-16.

24. Thampi P, Rao HV, Mitter SK, et al. The 5HT1a receptor agonist 8-Oh DPAT induces protection from lipofuscin accumulation and oxidative stress in the retinal pigment epithelium. PLoS One. 2012;7(4):e34468.

25. Wihlmark U, Wrigstad A, Roberg K, Brunk UT, Nilsson SE. Formation of lipofuscin in cultured retinal pigment epithelial cells exposed to pre-oxidized photoreceptor outer segments. APMIS. 1996;104(4):272-279.

26. Parinot C, Rieu Q, Chatagnon J, Finnemann SC, Nandrot EF. Large-scale purification of porcine or bovine photoreceptor outer segments for phagocytosis assays on retinal pigment epithelial cells. J Vis Exp. 2014;(94):52100.

27. Ran FA, Hsu PD, Wright J, Agarwala V, Scott DA, Zhang F. Genome engineering using the CRISPR-Cas9 system. Nat Protoc. 2013;8(11):2281-2308.

28. Lopes da Silva M, Cutler DF. von Willebrand factor multimerization and the polarity of secretory pathways in endothelial cells. Blood. 2016;128(2):277-285.

29. Baltazar GC, Guha S, Lu W, et al. Acidic nanoparticles are trafficked to lysosomes and restore an acidic lysosomal $\mathrm{pH}$ and degradative function to compromised ARPE-19 cells. PLoS One. 2012;7(12):e49635.

30. Guha S, Baltazar GC, Tu LA, et al. Stimulation of the D5 dopamine receptor acidifies the lysosomal $\mathrm{pH}$ of retinal pigmented epithelial cells and decreases accumulation of autofluorescent photoreceptor debris. J Neurochem. 2012;122(4):823-833.

31. Wavre-Shapton ST, Meschede IP, Seabra MC, Futter CE. Phagosome maturation during endosome interaction revealed by partial rhodopsin processing in retinal pigment epithelium. J Cell Sci. 2014;127(Pt 17):3852-3861.

32. Keeling E, Lotery AJ, Tumbarello DA, Ratnayaka JA. Impaired cargo clearance in the retinal pigment epithelium (RPE) underlies irreversible blinding diseases. Cells. 2018;7(2):16.

33. Keeling E, Culling AJ, Johnston DA, et al. An in-vitro cell model of intracellular protein aggregation provides insights into RPE stress associated with retinopathy. Int J Mol Sci. 2020;21(18):6647.
34. Agola JO, Hong L, Surviladze Z, et al. A competitive nucleotide binding inhibitor: in vitro characterization of Rab7 GTPase inhibition. ACS Chem Biol. 2012;7(6):10951108.

35. Guerra F, Bucci C. Multiple Roles of the Small GTPase Rab7. Cells. 2016;5(3):34.

36. Yoshimori T, Yamamoto A, Moriyama Y, Futai M, Tashiro Y. Bafilomycin A1, a specific inhibitor of vacuolar-type $\mathrm{H}(+)$-ATPase, inhibits acidification and protein degradation in lysosomes of cultured cells. J Biol Chem. 1991;266(26):17707-17712.

37. Zuhl AM, Nolan CE, Brodney MA, et al. Chemoproteomic profiling reveals that cathepsin $\mathrm{D}$ off-target activity drives ocular toxicity of beta-secretase inhibitors. Nat Commun. 2016;7:13042.

38. Rakoczy PE, Lai CM, Baines M, Di Grandi S, Fitton JH, Constable IJ. Modulation of cathepsin D activity in retinal pigment epithelial cells. Biochem J. 1997;324(Pt 3):935-940.

39. Rakoczy PE, Zhang D, Robertson $T$, et al. Progressive age-related changes similar to age-related macular degeneration in a transgenic mouse model. Am J Pathol. 2002;161(4):1515-1524.

40. Brunk UT, Wihlmark U, Wrigstad A, Roberg K, Nilsson SE. Accumulation of lipofuscin within retinal pigment epithelial cells results in enhanced sensitivity to photo-oxidation. Gerontology. 1995;41(Suppl 2):201-212.

41. Kim JY, Zhao H, Martinez J, et al. Noncanonical autophagy promotes the visual cycle. Cell. 2013;154(2):365-376.

42. Golestaneh N, Chu Y, Xiao YY, Stoleru GL, Theos AC. Dysfunctional autophagy in RPE, a contributing factor in age-related macular degeneration. Cell Death Dis. 2017;8(1):e2537.

43. Frost LS, Lopes VS, Bragin A, et al. The contribution of melanoregulin to microtubule-associated protein 1 light chain 3 (LC3) associated phagocytosis in retinal pigment epithelium. Mol Neurobiol. 2015;52(3):1135-1151.

44. Bright NA, Davis LJ, Luzio JP. Endolysosomes are the principal intracellular sites of acid hydrolase activity. Curr Biol. 2016;26(17):2233-2245.

45. Bassal M, Liu J, Jankowiak W, Saftig P, Bartsch U. Rapid and progressive loss of multiple retinal cell types in cathepsin D-deficient mice: an animal model of CLN10 disease. Cells. 2021;10(3):696.

46. Krohne TU, Stratmann NK, Kopitz J, Holz FG. Effects of lipid peroxidation products on lipofuscinogenesis and autophagy in human retinal pigment epithelial cells. Exp Eye Res. 2010;90(3):465-471. 